\title{
ASSISTÊNCIA HUMANIZADA DE ENFERMAGEM AO PACIENTE E A FAMÍLIA FRENTE AO DIAGNÓSTICO DE CÂNCER
}

\section{ARTIGO DE REVISÃO}

SILVA, Ariclenes dos Santos ${ }^{[1]}$, OLIVEIRA, Thais Carneiro Ferreira Silva [2], MAIA, Ana Margarete Cordeiro da Silva ${ }^{[3]}$

SILVA, Ariclenes dos Santos. OLIVEIRA, Thais Carneiro Ferreira Silva. MAIA, Ana Margarete Cordeiro da Silva. Assistência humanizada de enfermagem ao paciente e a família frente ao diagnóstico de câncer. Revista Científica Multidisciplinar Núcleo do Conhecimento. Ano 06, Ed. 03, Vol. 15, pp. 18-33. Março de 2021. ISSN: 2448-0959, Link de acesso:

https://www.nucleodoconhecimento.com.br/saude/assistencia-humanizada, DOI: 10.32749/nucleodoconhecimento.com.br/saude/assistencia-humanizada

\section{RESUMO}

Esse estudo teve como principal finalidade compreender como é realizada a assistência humanizada de enfermagem ao paciente e a família frente ao diagnóstico de câncer. Trata-se de uma pesquisa de revisão Bibliográfica do tipo descritiva com caráter qualitativo. A pesquisa foi realizada nas bases de dados da Scientific Electronic Library Online (Scielo), Literatura Latino-americana e do Caribe em Ciências da Saúde (Lilacs), Centro Latino-Americano e do Caribe de Informação em Ciências da Saúde (Bireme), Biblioteca Virtual de Saúde (BVS), e Bibliotecas Digitais de Universidades Brasileiras, por meio dos descritores: Humanização, Assistência de enfermagem, Paciente Oncológico. Para análise dos dados foi utilizada a análise de conteúdo de Bardin. Como resultado obteve-se as seguintes categorias: $\mathrm{O}$ impacto gerado pela neoplasia na vida da família; A importância do cuidado de enfermagem ao paciente com câncer; A humanização da assistência ao paciente e a família frente ao diagnóstico de câncer. Fica evidente, portanto, que o profissional de enfermagem frente ao paciente em tratamento de câncer precisa promover um cuidado 
humanizado considerado primordialmente ao paciente com câncer e a sua família envolvida na assistência, demonstrando sempre carinho, atenção e respeito individualizado para cada paciente em tratamento. Ademais, esses profissionais constituem-se como facilitadores desse processo, pela transmissão de confiança, cuidado e respeito ao paciente e sua família sendo o principal elo de apoio para o bem estar do paciente.

Palavras-chave: Humanização, Assistência de enfermagem, Paciente Oncológico.

\section{INTRODUÇÃO}

A palavra câncer teve origem no termo grego karkinos, que significa "caranguejo" sendo utilizado pela primeira vez por Hipócrates, o pai da medicina, que viveu entre 460 e 377 a.C. $O$ fato de ter sido descoberto em múmias egípcias comprova que o câncer é uma doença muito antiga por ter sido encontrada no homem há mais de três mil anos antes de cristo (INCA, 2019, p. 13).

O câncer é definido como um conjunto de mais de 100 doenças reunidas, que tem em comum a desorganização com crescimento irregular caracterizado por tumores malignos que invadem os tecidos e/ou órgãos e podem se espalhar pelo corpo. Vários fatores geralmente causados por agentes externos explicam a participação do câncer na mudança de perfil de adoecimento da população brasileira, entre eles podemos citar a maior exposição a agentes cancerígenos, sendo eles químicos, físicos ou biológicos (INCA, 2019).

Considerado como um grande problema de saúde pública, principalmente em países em desenvolvimento, por ter uma incidência nacional e ser de perfil epidemiológico elevado, tendo grande custo para o governo. A mais recente estimativa mundial mostra que, em 2018, ocorreram 18 milhões de casos novos de câncer. Para o Brasil, a estimativa para cada ano do triênio 2020-2022, aponta que seja de 625 mil casos novos de câncer (INCA, 2019, p. 25). 
Apesar das estimativas de casos novos serem altas, as chances de cura aumentaram devido aos avanços tecnológicos, estudos clínicos elevados, atendimento multidisciplinar, opções de escolhas de novas drogas, assistência humanizada ao paciente e seus familiares. No entanto nem sempre se tem a cura e nesse momento é essencial que os pacientes e familiares passem a serem avaliados, e necessitem de cuidados dos profissionais de enfermagem que vão atuar no controle e alivio da dor física, além do acompanhamento psicológico para os aspectos sociais e espirituais visando assim uma melhor qualidade de vida para o indivíduo (BESERRA; AGUIAR, 2020).

Visto que os profissionais de enfermagem está a linha de frente do processo saúdedoença, e nos momentos de total sofrimento e obrigação, quanto as emoções a o processo do trabalho, não se deve criar a antiga postura de não se envolver, visando que $o$ ato de cuidar de um paciente dentro de um tratamento intensivo como o do câncer, precisa muito mais de apenas conhecimentos científicos. Aborda-se de uma demonstração de carinho, e sobretudo de uma humanização assistencial. Assim, mostra que é importante criar laços afetivos durante o tratamento, entre os profissionais de enfermagem, paciente e familiares (SILVA, 2015).

Apesar de todos os avanços científicos e tecnológicos o câncer ainda causa o estigma do adoecer e da morte no paciente que receber o diagnóstico, o que enfrentam impactos importantes sobre todos que estão à sua volta. Quando o câncer é diagnosticado, a percepção da finitude da vida torna-se presente é normal que apareçam mitos e fantasias em torno da doença e de seu tratamento. Nessa perspectiva, a contribuição da enfermagem torna-se fundamental, uma vez que oferece uma assistência integral e humanizada, contribuindo para o bem-estar do paciente por meio da reabilitação e prestação do cuidado que visam aliviar os impactos causados pela doença (BESERRA; AGUIAR, 2020).

Nesse contexto, o processo de Humanização do cuidar surge na atualidade para que se possa repensar as ações praticadas todos os dias, reformulando o atendimento que se vem prestando ao paciente. Dessa forma, é importante abordar a necessidade 
da humanização do cuidado de Enfermagem, para estimular uma reflexão da equipe em relação aos cuidados prestados aos pacientes com câncer e sua família em uma unidade oncológica (DUARTE; NORO, 2010).

Considerando que o cuidar é uma ação fundamental para a promoção e recuperação da saúde no cuidado aos pacientes oncológicos, é necessário que os profissionais de enfermagem atuem em todos os níveis de atenção, tornando essencial possuir o conhecimento técnico-científico e habilidades entre a equipe, favorecendo ao paciente em tratamento ações de forma integrais, participativas e resolutivas, na recuperação do paciente (CARDOSO; SANTOS; MORGADO, 2017).

Entende-se que o cuidar é fundamental na recuperação e promoção da saúde do paciente, exigindo um atendimento de alta qualidade e de extrema eficiência para um profissional de enfermagem, desta forma, a atuação do enfermeiro (a) em domicilio ou ambiente hospitalar, torna-se essencial, especialmente, em pessoas com uma neoplasia, tendo em vista que estes indivíduos por receber o diagnóstico do câncer tem uma expectativa de vida e cura fragilizadas, necessitando do cuidado e da atenção (RODRIGUES; FERREIRA, 2011).

O cuidado de enfermagem ao paciente com câncer deve-se manifestar em atitudes que valorizem e engrandeça o ser humano, o respeito ao próximo é fundamental, assim as relações entre profissionais e pacientes depende de um compromisso individual. O cuidado deve estar pautado essencialmente na construção de valores éticos e morais, respeitando os saberes e as crenças de cada pessoa (SILVA; JESUS; BORGES, 2015).

A Oncologia por ser um ramo da ciência que trabalha com tumores e com o câncer desde o seu diagnóstico, intervenções e os diferenciados tipos de tratamento, é necessário o desenvolvimento de cuidados na atenção ao paciente e a família, estabelecendo grandes desafios para os profissionais de enfermagem a fim de prestar uma assistência de forma mais humanizada possível, tanto para o paciente quanto para a família. Visto que os profissionais de saúde, em especial da enfermagem 
precisa ter um olhar criterioso no desenvolvimento das práticas e ações que serão ofertadas para os pacientes oncológicos, o que irá favorecer uma assistência humanizada e segura. Logo, esse estudo teve como objetivo, compreender como é realizada a assistência humanizada de enfermagem ao paciente e a família frente ao diagnóstico de câncer.

\section{METODOLOGIA}

Trata-se de uma pesquisa de revisão Bibliográfica do tipo descritiva com caráter qualitativa. De acordo com Gil (2007), a pesquisa bibliográfica é desenvolvida com base em material já elaborado, constituído principalmente de livros e artigos científicos, ou seja, é aquela que apresenta o que diversos autores relatam sobre um determinado fenômeno ou objeto analisado, contribuindo para se entender melhor suas características e o que vem sendo compartilhado pela comunidade científica.

Para guiar a revisão, formulou-se a seguinte questão norteadora: Como é realizada a assistência humanizada de enfermagem ao paciente e a família frente ao diagnóstico de câncer? Para seleção da amostra foi utilizado os artigos científicos produzidos no período de 2010 a 2020. Os bancos de dados utilizados foram: Scientific Electronic Library Online (Scielo), Literatura Latino-americana e do Caribe em Ciências da Saúde (Lilacs), Centro Latino-Americano e do Caribe de Informação em Ciências da Saúde (BIREME), Biblioteca Virtual em Saúde (BVS), e Bibliotecas digitais de universidades brasileiras.

Como critérios de inclusão para a pesquisa foram: Artigos escritos em português de 2010 a junho de 2020, autores da área de enfermagem e que atenderam os objetivos da pesquisa. Já os critérios de exclusão foram: artigos que não foram publicados em língua nacional, que não estavam disponíveis na integra e que não estavam dentro do objeto proposto pela pesquisa.

A coleta de dados foi realizada a partir de publicações dos anos do referido período indexado nas bases de dados já citadas, utilizando-se as palavras-chave: 
Assistência humanizada, cuidado de enfermagem ao paciente com câncer, o impacto gerado pela neoplasia na vida das famílias.

Para análise dos dados foi utilizada a análise de conteúdo de Bardin (2011), onde os dados puderam ser trabalhados em três etapas básicas: Na Pré-análise o material foi organizado e feito uma leitura minuciosa. Na exploração do Material todo o material foi submetido a um estudo aprofundado, utilizando-se procedimentos como a codificação e a categorização dos dados. E por fim foi feito o Tratamentos dos resultados obtidos e interpretação, nesta fase aprofundou-se a análise dos dados tratando de desvendar o conteúdo latente que eles possuíam, onde foi feita a redação final.

\section{RESULTADOS / DISCUSSÃO}

$\mathrm{Na}$ busca realizada em todas as bases de dados referidas, foram identificados inicialmente 25 artigos e no fim do processo de seleção, foram identificados 13 (treze) artigos, os quais cumpriram todos os critérios de elegibilidade pré-estabelecidos, e foram, portanto, incluídos nessa revisão. Dos 13 artigos selecionados, 07 (sete) são pesquisas de revisão integrativa, duas do tipo descritivas, três qualitativa e um quantitativo.

Após analisarmos as produções que foram selecionadas, os resultados foram agrupados em três categorias a seguir: $O$ impacto gerado pela neoplasia na vida da família; A importância do cuidado de enfermagem ao paciente com câncer; e A humanização da assistência ao paciente e a família frente ao diagnóstico de câncer.

\section{O IMPACTO GERADO PELA NEOPLASIA NA VIDA DA FAMILIA}

O aumento significativo das neoplasias malignas faz com que aconteça o crescimento desorganizado das células, que por sua vez podem invadir tecidos e órgãos, essa desorganização permite que as células se dividam rapidamente, sendo muito agressivas e incontroláveis, formando tumores, que podem se disseminar por todas as partes do corpo (INCA, 2019). 
Segundo Herco, et al., (2014) refere que a neoplasia faz parte de uma área que manifesta uma aproximação no processo morte-morrer, por isso é necessário que se tenha cuidados de alta complexidade. Por ser uma doença crônica, ela traz efeitos indesejáveis para o paciente, afetando assim sua autoestima, e seu aspecto psicossocial (SANTOS; LATTARO; ALMEIDA, 2011).

Há diversidades de tipos de câncer que podem coincidir com variados tipos de células do corpo. Podendo começar em tecidos epiteliais ou conjuntivos, sendo os epiteliais denominados carcinomas, que condiz a pele e mucosas, ou se partir de tecidos conjuntivos, como ossos, cartilagem e músculos, sendo chamados de sarcomas, ainda, há outros sinais que diferenciam outros tipos, como a velocidade de multiplicação das células, e a facilidade de invadir órgãos vizinhos (INCA,2019).

Essa doença surge quando há uma mutação genética (alteração no DNA da célula), onde se tem comando errados para realizar suas atividades. Essas alterações podem ocorrer em genes especiais, chamados de proto-oncogenes que estão paralisados em células normais. Quando há a ativação dessas células, o proto-oncogenes, passam ser oncogênese, transformando assim, as células em cancerosas (INCA, 2019).

Segundo Benedetti, et al. (2014), a família configura-se, portanto, como essencial no processo de suporte do paciente com câncer que se une e se disponibiliza para auxiliar o doente, principalmente porque a doença não é um problema a ser enfrentado somente pelo indivíduo, mas também pelos seus familiares, amigos e pessoas do seu convívio, causando sérios impactos, apesar de recursos oferecidos, ainda pode notar a falta de informação e preconceitos que impedem o diagnóstico e tratamento precoce do paciente. Toda a família deve estar unida em busca de um objetivo comum, participar no cuidado a ser prestado, dar apoio emocional, e ajudar em tudo que for possível para ajudar o doente a superar seus temores diante o diagnóstico e tratamento do câncer. 


\section{A IMPORTÂNCIA DO CUIDADO DE ENFERMAGEM AO PACIENTE COM CÂNCER}

A Sistematização da Assistência de Enfermagem (SAE) é um instrumento de grande importância uma vez que auxilia o profissional de enfermagem no desenvolvimento de suas ações prestadas ao paciente com neoplasia, através de ferramenta que possibilita uma maior visibilidade no trabalho do profissional de enfermagem de acordo com o método científico e o referencial teórico, de modo que seja possível que os profissionais de enfermagem identifiquem as necessidades humanas básicas prestando o melhor atendimento ao paciente durante o tratamento do câncer, família e pela aplicação das fases que compõem o processo de enfermagem, sendo elas: histórico de enfermagem, diagnóstico de enfermagem, planejamento, implementação e avaliação, possibilitando ao enfermeiro o desenvolvimento de um cuidado holístico, atendendo às especificidades e singularidades de cada paciente (SILVA; MOREIRA; 2011).

O SAE ao paciente com câncer é realizado através do Processo de Enfermagem, é um instrumento importante que orienta e viabiliza a trabalho do profissional da enfermagem, pois, na sua implementação, pode refletir na qualidade dos cuidados de enfermagem ao paciente oncológico, além de permitir independência da equipe de enfermagem (NASCIMENTO, 2012).

O cuidar exige um processo de alta dedicação, compreensão e paciência, e esse cuidado deve ser, dos profissionais de saúde, quanto mesmo de sua família, apesar dos profissionais ser muito maior, pelo um conhecimento mais amplo sobre a patologia envolvida. A enfermagem pode passar uma orientação da primeira notícia, mas para isso é importante que se tenha um planejamento da assistência de como manter um diálogo com familiares e o paciente, como também trabalhar o emocional de cada um, de forma verdadeira, firme, e com certa sensibilidade, no intuito de diminuir a angústia da família e do paciente em tratamento, e assim restabelecer a condição de vida de todos os envolvidos durante o tratamento promovendo o conforto e segurança. Prestar assistência a pacientes com câncer é lidar com o ser humano e com suas 
necessidades básicas, portanto, considera-se que estes pacientes em tratamento são dignos de uma assistência integral e um olhar mais humanizado durante o tratamento para promover melhor recuperação do doente e prestar aos familiares acolhimento já que o câncer causa impacto em todos. (BARBOSA et al., 2019).

O paciente com câncer é um indivíduo vulnerável a adversidades de caráter psicológico, biológico, social ou espiritual que podem ser desencadeadas desde do recebimento do diagnóstico de câncer e efeitos colaterais dos diversos meio de tratamento existente. Nessa perspectiva o enfermeiro é orientado a compreender o paciente visando às demandas geradas no processo assistencial, considerando as necessidades individuais de cada paciente. Nesse sentido, o enfermeiro(a) contribui para a manutenção do paciente e da família, ajudando no enfrentamento ao estresse ocasionado pela doença, durante o tratamento ao até mesmo quando todos os recursos terapêuticos forem esgotados procurando garantir uma assistência integral ao paciente e família. Por tanto, para que tenha qualidade das ações, os cuidados de saúde dados, requer atenção da equipe de enfermagem situado no paciente com câncer, o que exige habilidades específicas as quais não devem estar restritas aos cuidados direcionados à dor e ao sofrimento, porém extensivo aos familiares, para que se tenha melhor interação entre o paciente e sua família, e buscar identificar e auxiliar as necessidades do paciente e proporcionar a saúde com olhar na dimensão biopsicossocial. (RIBEIRO et al., 2016).

Os cuidados devem estar voltados de maneira integral para o paciente e família, de forma holística, humanizada, priorizando a comunicação para o tratamento adequada, para promover o afeto, carinho e atenção de cada indivíduo. O cuidar vai além das ações terapêuticas, consiste em confortar o paciente, requer criação de vínculo, saber ouvir, compreender o sofrimento. O relacionamento entre enfermeiro(a), paciente e família deve atingir um sentido mais amplo, exigindo comunicação, compreensão e aceitação entre a equipe de enfermagem, fazendo com que a visão holística e a humanização no cuidar sejam eficientes nesta área tão complexa, para, assim, promover uma assistência de qualidade, mantendo uma relação de confiança entre todos envolvidos no tratamento do paciente com câncer. (SANTOS et al., 2018). 


\section{A HUMANIZAÇÃO DA ASSISTÊNCIA AO PACIENTE E A FAMÍLIA FRENTE AO DIAGNÓSTICO DE CÂNCER}

No Brasil, em 1993 foi instituído o Programa de Humanização da Assistência Hospitalar que possuiu como pilares, agregar a competência e métodos técnicos e cientifica a aspectos ética que compreendesse a individualidades das necessidades do paciente e do profissional de saúde, respeitando os limites e necessidades peculiares de cada um e a convivência com o incógnito e o imprevisível que corrobora para um tratamento digno de cada indivíduo (BARBOSA et al., 2013).

Em 2003 para fortalecer a prática diária da humanização do paciente, o Ministério da Saúde desenvolveu a Política Nacional de Humanização intervindo e colocando em ação os princípios norteadores base do Sistema Único de Saúde, que possui como fundamentação teóricos e metodológicos visando fortalecer as políticas do SUS tendo em vista a atenção e reorganização da gestão dos serviços de saúde suscitando transformações nos modos de gerir e prestar assistência pautada na qualidade aos usuários fomentando agregar mais ainda o sistema da saúde pública no Brasil e promovendo as relações e comunicação entres os gestores, trabalhadores de saúde e usuários abrangendo a todos. Humanização se traduz, como inserção das diferenças nos métodos de gestão e da assistência humanizada de enfermagem ao paciente, família e demais acompanhantes na busca por melhor aproximação e empatia por todos. E essas mudanças são compostas não por um indivíduo ou grupo separado, mas de forma coletiva e através do compartilhamento dos diferentes saberes para a recuperação do paciente em tratamento da neoplasia, incluir para estimular novas tecnologias e promover uma assistência mais organizada para prestar melhor atendimento ao paciente que muitas das vezes está em situação de fragilidade por conta do tratamento contra o câncer (BRASIL, 2015).

O cuidado de enfermagem humanizado à pessoa com câncer, tende prover uma assistência diferenciada, conforto, agir e reagir devidamente frente ao paciente com câncer, família e consigo mesmo; proporcionar crescimento pessoal do paciente com câncer, da família e de si mesmo, valorizar o sofrimento e as conquistas, empodera 
o próximo com seu cuidado e empoderar-se pelo cuidado prestado, empenhar-se para preservar a integridade física, moral, emocional e espiritual, agregar-se, vincularse e apoiar o outro e a si mesmo a encontrar significados nas situações, pois os pacientes com câncer carregam, com a doença o estigma da incerteza de cura, o medo da morte, a depressão e a ansiedade, mas também a luta e a vontade de viver (PIMENTA, 2010).

O cuidado humanizado de enfermagem pode ser entendido como a percepção do significado da vida e a capacidade de distinguir e compreender a si mesmo e ao outro. Ainda, realizar na prática de cuidar à compreensão da pessoa em sua peculiaridade e em sua originalidade de ser. Nesse sentido, a assistência de enfermagem a pacientes com câncer envolve empatia respeito ao próximo, às suas limitações, à dor e ao sofrimento humano, à perseverança, à vida e a morte. Desta forma, pertence ao enfermeiro (a) oncológica procurar desenvolver os seus conhecimentos com fundamentação técnico científica e assistencial, atividades de caráter educativo, relacionadas à prevenção, detecção precoce, assistência e reabilitação, buscando junto à equipe multidisciplinar, paciente e família empenhada no tratamento e recuperação do paciente com a neoplasia, para melhorar no campo de atuação práticas aos cuidados dos pacientes com câncer. Adquirindo o interesse de se atualizar os seus conhecimentos na área oncológica, e dessa maneira aprimorar a qualidade do atendimento assistencial e humanizado prestados aos pacientes com câncer. Portanto, o enfermeiro deve possuir além de aptidões técnicas, compreensão e sensibilidade para proporcionar uma assistência ética e humanizada pautada às reais necessidades do paciente em cada estágio do tratamento, considerando essas individualidades, enxergando-o como protagonista no planeamento da assistência, preservando seu direito de escolha. (SOUSA et al., 2017).

O diagnóstico de câncer é um impacto chocante e constante, devido à ameaça de morte, e que pode despertar no indivíduo e em seus familiares reações adversar e sentimentos, assim é importante o resultado do diagnóstico e o início do tratamento quanto antes para diminuir os efeitos ao paciente. A família sofre impacto significativo quando um membro recebe o diagnóstico de câncer, esse deve ser reconhecido pelo 
enfermeiro(a), para melhorar a assistência prestada e inserir a família no plano de cuidado da equipe de enfermagem. E assim, quando a família se torna incapaz de lidar com as questões do tratamento do paciente com câncer ou quando a mesma precisa de apoio para suportar os problemas e dificuldades da doença, como crises de dor, ou as reações adversas advinda da quimioterapia, surge a enfermagem, que presta assistência não só o paciente, mas também à família, a qual acaba por ser esquecida em sua dor, (OLIVEIRA et al. ,2012).

Nesse contexto é que o profissional de enfermagem domina uma ferramenta singular que pode ter mais influência sobre o paciente do que qualquer fármaco ou tratamento. Para tal, faz-se imprescindível uma autoanálise que constitui uma ferramenta essencial de todas as ações, influi na qualidade da assistência prestada aos pacientes que necessitam dos cuidados de enfermagem. O enfermeiro deve estar pronto para dar suporte e assistência não somente ao paciente oncológico, mas também a seus familiares acolhendo nas diversidades de crise físicas, emocionais, culturais, financeiras e espirituais assim promovendo um apoio desde o diagnostico passando pelo tratamento chegando à cura ou a morte (ALVES, 2011).

Assim é relevante considerar que atender pessoas corresponde a prestar cuidados a um público com suas singularidades. Além disso, possibilita durante o tratamento o desenvolvimento de forma mais humana, considerando o paciente com câncer como um ser individualizado e de particularidades próprias sejam elas físicas e/ ou emocionais. Assim o enfermeiro(a) precisa adquirir uma visão holística e humanizada, na busca de compreender que, nas diferentes relações desenvolvidas, é preciso estabelecer e proporcionar uma abordagem profissional humana, contribuindo para não só o processo de saúde, mas principalmente, de vida. (SOUSA, et al., 2017).

\section{CONCLUSÃO}

Com a elaboração do presente estudo evidenciou a importância da assistência humanizada de enfermagem ao paciente com neoplasia e a família uma vez que os tratamentos direcionados ao câncer suscita importantes repercussões físicas, 
psicológicas e financeiras, tendo em vista a magnitude e o estigma causado pelo câncer ao paciente. Neste contexto, a atuação humanizada do enfermeiro (a) tem o objetivo de contribuir para o alívio do sofrimento do paciente, bem como promover um atendimento humanizado digno e de direito para o ser humano.

Deste modo, compreender de forma holística cada paciente considerando sua complexidade, mudanças emocionais, e efeitos das sessões de quimioterapia e radioterapia, uma vez que cada paciente exige além de cuidados básicos de enfermagem, maior apoio e atenção por meio de um relacionamento de confiança e respeito, mediado por meio de dialogo claro e de um atendimento humanizado e objetivo, com a necessidade de comunicação constante entre paciente e família, desenvolvendo a empatia e deixando o paciente e a família mais acolhida para enfrentar a doença.

Evidenciou-se neste estudo que a Sistematização da Assistência de Enfermagem (SAE), é de grande importância para os profissionais de enfermagem, pois, permite prestar uma assistência organizada com qualidade e humanizada, auxiliando o enfermeiro (a) na tomada de decisões, avaliando as consequências, além de identificar as melhorias no cuidado do paciente com câncer.

Dessa forma, a assistência humanizada de enfermagem prestada a um paciente em tratamento de câncer, poderá propiciar melhor qualidade de vida pelo tempo em que estiver na hospitalização. Ademais, a humanização de enfermagem promove mudanças no ambiente familiar e do profissional. Enfim, a assistência de enfermagem frente ao paciente com câncer que estão fragilizados fisicamente e emocionalmente precisa ser ética e humanizada.

Fica, portanto o aprendizado que cabe ao profissional de enfermagem qualificar-se no âmbito da prestação de serviços ao paciente oncológico, uma vez que se trata de um grupo de pessoas que exigem além dos cuidados básicos de enfermagem, maior apoio, atenção e atendimento direcionado de acordo com as individualidades de cada paciente. 


\section{REFERÊNCIAS}

ALVES, Vanessa Souza et al. Conhecimento de Profissionais da Enfermagem sobre Fatores que Agravam e Aliviam a Dor Oncológica: Revista Brasileira de Cancerologia v. 57, n.2, pp. 199-206. Maceió, 2011. Disponível em: https://rbc.inca.gov.br/revista/index.php/revista/article/view/706. Acesso em: 20 de Mar. 2020.

BARBOSA, Guilherme Correa et al. Política nacional de humanização e formação dos profissionais de saúde: revisão integrativa. Revista Brasileira de Enfermagem. V. 66, n.1, 123-7. Brasília-DF, 2013. Disponível em: https://www.scielo.br/scielo.php?pid=s0034-71672013000100019\&script=sci_arttext. Acesso em: 20 de Mar. 2020.

BARBOSA, Andréa Nunes et al. A importância da assistência humanizada prestada pelo enfermeiro nos cuidados paliativos ao paciente oncológico terminal: Revista Brasileira Interdisciplinar de Saúde. v.1, n.4, p. 92-6, Brasília-D, 2019.Disponível em: http://revista.rebis.com.br/index.php/rebis/article/view/248. Acesso em: 18 de Mar. 2020.

BARDIN, L. Analise de conteúdo; tradução Luís Antero Reto, Augusto Pinheiro São Paulo - SP; Edição 70, 2011.

BENEDETTI, Gabriella Michel dos Santos et al. O tratamento do câncer infanto juvenil: desvelando as vivências dos pais: Revista Latino-Americana de Enfermagem, v 22 n3, p. 452-31, Mirador -PR, 2014. Disponível em: https://www.scielo.br/scielo.php?pid=S010411692014000300425\&script=sci_arttext\& tlng=pt. Acesso em: 20 de Jan. 2020.

BESERRA, JHGN; AGUIAR, RS. Sentimentos vivenciados pela equipe de enfermagem perante o tratamento de pacientes com câncer: revisão integrativa. Revista Revisa. v.9, n 1.p.144-55. Brasília-DF,2020. Disponível 
em: http://revistafacesa.senaaires.com.br/index.php/revisa/article/view/485. Acesso em: 20 de Jan. 2020.

BRASIL, RESOLUÇÃO no 466, de 12 de dezembro de 2012. Dispõe sobre diretrizes e normas regulamentadoras das pesquisas envolvendo seres humanos. Brasília, 2012.

Disponível

em:

http://www.conselho.saude.gov.br/resolucoes/2012/Reso466.pdf. Acesso em: 18 de Mar. 2020.

BRASIL. Ministério da Saúde (MS). Política Nacional de Humanização- PNH. Biblioteca Virtual em Saúde do Ministério da Saúde, Brasília, 2015. Disponível em: http://bvsms.saude.gov.br/bvs/folder/politica nacional humanizacaopnh 1ed.pdf. Acesso em: 30 de Mar. 2020.

CARDOSO, Juliana Abreu; SANTOS, Maria Natividade Pereira; MORGADO, Sara Santos Mello. Atuação do enfermeiro no cuidado do paciente oncológico no domicílio. Revista Eletrônica Atualiza Saúde, v. 6, n. 6, p. 36-42, Salvador- BA, 2017. Disponível em: http://atualizarevista.com.br/wpcontent/uploads/2017/08/Atua\%C3\%A7\%C3\%A3o-do-enfermeiro-no-cuidado-dopaciente-oncol\%C3\%B3gico-no-domic\%C3\%ADlio-v-6-n-6.pdf. Acesso em: 06 Out. 2019.

DUARTE Maria Lourdes Custódio; NORO, Adelita. HUMANIZAÇÃO: uma leitura a partir da compreensão dos profissionais da enfermagem. Revista Gaúcha Enfermagem, v.31 n.4, Porto Alegre- RS, 2010. Disponível em: https://www.scielo.br/scielo.php?script=sci_arttext\&pid=\$198314472010000400011\& tlng=pt. Acesso em: 06 de Out. 2019.

HERCOS, Thaís Machado et al. O trabalho dos profissionais de enfermagem em unidades de terapia intensiva na assistência ao paciente oncológico: Revista Brasileira de Cancerologia, v 60, n.1 p. 51-58, Ribeirão Preto-SP,2014. Disponível em: https://rbc.inca.gov.br/revista/index.php/revista/article/view/495. Acesso em: 04 Out. 2019. 
INCA. INSTITUTO NACIONAL DE CÂNCER (BRASIL). ABC DO CÂNCER: abordagens básicas para o controle do câncer / Instituto Nacional de Câncer. Rio de Janeiro: Inca, 2019. Disponível em: https://www.inca.gov.br/sites/ufu.sti.inca.local/files//media/document//ivro-abc-5edicao.pdf. Acesso em: 21 de Mar. 2020.

INCA. INSTITUTO NACIONAL DE CÂNCER (BRASIL). Estimativa 2020: Incidência de câncer no Brasil / Instituto Nacional de Câncer. Rio de Janeiro: Inca, 2019. Disponível em: https://www.inca.gov.br/sites/ufu.sti.inca.local/files//media/document/estimativa2020-incidencia-de-cancer-no-brasil.pdf. Acesso em: 21 de Set. 2019.

INCA. INSTITUTO NACIONAL DE CÂNCER (BRASIL). O que é o câncer? / Instituto Nacional de Câncer. Rio de Janeiro: Inca, 2019. Disponível em: http://www.inca.gov.br/o-que-e-cancer. Acesso em: 21 de Mar. 2020.

NASCIMENTO, Luzia Kelly Alves Silva et al. Sistematização da assistência de enfermagem a pacientes oncológicos: uma revisão integrativa da literatura. Revista Gaúcha de Enfermagem, v.33, n.1: p. 177-85. Porto Alegre- RS, 2012. Disponível em:

https://www.scielo.br/scielo.php?pid=S198314472012000100023\&script=sci_abstract \&tIng=es. Acesso em: 20 de Out. 2019.

OLIVEIRA, William Tiago et al. Vivência do cuidador familiar na prática do cuidado domiciliar ao doente crônico dependente. Revista Ciênccuid saúde, v. 11.n. 1: p.129-137.Maringá, 2012.Disponível em: http://periodicos.uem.br/ojs/index.php/CiencCuidSaude/article/view/18869. Acesso em: 26 de Out.2020.

PIMENTA, Cibele Andrucioli Mattos. Cuidados paliativos: uma nova especialidade do trabalho da enfermagem? Acta Paulista de Enfermagem, v. 23, n. 3, pp. 7-8, São Paulo, 2010. Disponível em: https://www.scielo.br/scielo.php?pid=S0103$21002010000300001 \&$ script=sci_arttext. Acesso em: 09 de Fev. 2020. 
RIBEIRO, Juliane Portella et al. Assistência de enfermagem ao paciente oncológico hospitalizado: diagnósticos e intervenções relacionadas às necessidades psicossociais e psicoespirituais. Revista pesqui. cuid. Fundam. V8, n.4 p. 51365142. Rio de Janeiro, $2016 . \quad$ Disponível em: http://www.seer.unirio.br/index.php/cuidadofundamental/article/viewFile/4016/pdf. Acesso em: 09 de Fev. 2020.

RODRIGUES, Juliana Stoppa Menezes; FERREIRA Noeli Marchioro Liston Andrade. A experiência da família no cuidado domiciliário ao doente com câncer: uma revisão integrativa. Revista Eletrônica de Enfermagem, v 13 n 2, 338-46. UFSCAR. São Carlos - SP,2011. Disponível em: https://www.revistas.ufg.br/fen/article/view/8980. Acesso em: 10 de Fev. 2020.

SANTOS, Demétria Beatriz Alvarenga; LATTARO, Renusa Campos Costa; ALMEIDA, Denize Alves. Cuidados paliativos de enfermagem ao paciente oncológico terminal: revisão da literatura. Revista de Iniciação Científica da Libertas, v.1, n.1, p.72-84, São Sebastião do Paraiso,2011. Disponível em: http://www.libertas.edu.br/revistas/index.php/riclibertas/article/view/14. Acesso em: 28 de Nov.2019.

SANTOS, Alda Laisse Nascimento; LIRA, Sabrina Souza; COSTA Ruth Silva Lima. Cuidados paliativos prestados pelo enfermeiro ao paciente oncológico: Revista de Ciências em Foco, v.2, n.1, p. 63-73, Rio Branco,2018. Disponível em: http://revistas.uninorteac.com.br/index.php/DeCienciaemFoco0/article/view/147. Acesso em: 28 de Nov. 2019.

SILVA, Adriana Ferreira. et al. Cuidados paliativos em oncologia pediátrica: percepções, saberes e práticas na perspectiva da equipe multiprofissional. Revista Gaúcha de Enfermagem, v.36, n.2, p. 56-62, Porto Alegre - RS, 2015. Disponível em: https://www.lume.ufrgs.br/handle/10183/129504. Acesso em: 29 de Nov. 2019.

SILVA, Helen Cristina Soares; JESUS; Maria Helena; BORGES, Rodrigo Câmara. A importância da equipe de enfermagem no acolhimento aos familiares de 
pacientes oncológicos. ICESP/Promove de Brasília. Disponível em: http://www.nippromove.hospedagemdesites.ws/anais.simposio/arquivos up/documentos/552ad8fa3edabb181adb4a1b2bddaf2f.pdf. Acesso em: 29 de Mar. 2020.

SILVA, Marcelle Miranda; MOREIRA, Marléa Chagas. Sistematização da assistência de enfermagem em cuidados paliativos na oncologia: visão dos enfermeiros: Acta Paulista de Enfermagem, v. 24, n. 2, pp. 172-8, São Paulo, 2011. Disponível em: https://www.scielo.br/scielo.php?pid=S0103-21002011000200003\&script=sci_arttext. Acesso em: 28 de Mar. 2020.

SOUSA, Joyce Caroline Oliveira; SOUSA, Caíque Rodrigues Carvalho. A importância de um atendimento humanizado no tratamento do paciente oncológico: Revista Científica Multidisciplinar Núcleo do Conhecimento. v.5, n.9, pp. 126-141, Teresina, 2017.Disponível em: https://www.nucleodoconhecimento.com.br/saude/tratamento-do-pacienteoncologico?format=pdf. Acesso em 30 de Mar. 2020.

Enviado: Março, 2021.

Aprovado: Março, 2021. 